\title{
New Modified Pekkari Model to Analyse the Aeroelastic Stability Behaviour for a Flexible Overexpanded Rocket Nozzle
}

\author{
N. Bekka, M. Sellam, and A. Chpoun
}

\section{Introduction}

During the transient phases, start-up and shutdown of the rocket engines, or when it operates under no adaptation conditions at low altitude, very complex physical phenomena appear, particularly the aeroelastic effects due to extended boundary layer separation and its interaction with the shock wave in the interior of the divergent of the nozzle. The numerical coupling strategy to study this phenomenon using multi-physics codes is considered to be the most used technique today [1-7]. Knowing that the present study is limited to the analysis of linear stability relative to the small displacements, the choice of the transpiration technique to consider the coupling at the fluid-structure interface is justified by its simple implementation and its low CPU time computation compared to those of the ALE (arbitrary Lagrangian-Eulerian) method, widely discussed in many previous studies.

The fluid dynamic solver used for solving the Euler equations for a 2D inviscid compressible flow case is the NSC2KE code developed at INRIA by Mohammadi [8]. The code uses a combination of the finite volume and finite element methods to simulate perfect or viscous flows around or inside $2 \mathrm{D}$ or axisymmetric configurations on unstructured meshes. The structure dynamic solver is based on the implicit Newmark scheme to describe the temporal evolution of the structure displacements. For the coupling technique and for temporal integration, unlike to the code of structure, the NSC2KE fluid code is based on an explicit scheme in which the fluid time step is conditioned by the CFL stability condition. This difficulty leads to a time shift between the two codes, which can be overcome by using the iterative scheme CSS (conventional serial staggered

N. Bekka $(\bowtie) \bullet$ M. Sellam • A. Chpoun

Laboratoire de Mécanique et d'Energétique d'Evry LMEE, Université d'Evry Val d'Essonne, 40 rue du Pelvoux, 91020 Evry Cedex, France e-mail: Nadir.Bekka@ufrst.univ-evry.fr procedure) [9]. To validate the numerical coupling procedure, the main results obtained for a basic $2 \mathrm{D}$ configuration of an overexpanded rocket nozzle are compared with those of the aeroelastic stability models developed in previous works for the inviscid flow case [1].

\section{Numerical Method}

As mentioned previously, the simulation of aeroelastic phenomena is done by coupling two numerical codes. The first one is for the fluid dynamics part to resolve the Euler equations using the conservative formulation for a $2 \mathrm{D}$ compressible inviscid flow:

$$
\frac{\partial U}{\partial t}+\frac{\partial F(U)}{\partial x}+\frac{\partial G(U)}{\partial y}=0
$$

where $U, F(U)$ and $G(U)$ are respectively the vector of the conservative variables and the components of the convective flux:

$$
\begin{gathered}
U=\left(\begin{array}{c}
\rho \\
\rho u \\
\rho v \\
\rho E
\end{array}\right) \\
F(U)=\left(\begin{array}{c}
\rho u \\
\rho u^{2}+p \\
\rho u v \\
(\rho E+p) u
\end{array}\right), \quad F(U)=\left(\begin{array}{c}
\rho v \\
\rho u v \\
\rho v^{2}+p \\
(\rho E+p) v
\end{array}\right)
\end{gathered}
$$

where $\rho$ is the mass density, $\vec{u}=(u, v)$ the velocity, $E$ the total energy and $p=\rho(\gamma-1)\left(E-\frac{u^{2}+v^{2}}{2}\right)$ the pressure.

The time integration is achieved by using an explicit Runge-Kutta scheme of order 4, and the time step is determined by the CFL criterion. 
The second code is dedicated to the structure computations using the implicit Newmark scheme to describe the temporal evolution of the structure displacements:

$$
\begin{aligned}
& \left\{W^{p+1}\right\}=\left\{W^{p}\right\}+\Delta t\left\{\dot{W}^{p}\right\}+\frac{\Delta t}{2}\left((1-b)\left\{\ddot{W}^{p}\right\}+b\left\{\ddot{W}^{p+1}\right\}\right) \\
& \left\{\dot{W}^{p+1}\right\}=\left\{\dot{W}^{p}\right\}+\Delta t\left((1-a)\left\{\ddot{W}^{p}\right\}+a\left\{\ddot{W}^{p+1}\right\}\right) \\
& \left\{\ddot{W}^{p+1}\right\}=\frac{4}{\Delta t^{2}}\left(\left\{W^{p+1}\right\}-\left\{W^{p}\right\}\right)-\frac{4}{\Delta t}\left\{\dot{W}^{p}\right\}-\left\{\ddot{W}^{p}\right\}
\end{aligned}
$$

In this scheme, the constants $a$ and $b$ are set equal to 0.5 .

$\left\{W^{p}\right\}, \quad\left\{\dot{W}^{p}\right\}$ and $\left\{\ddot{W}^{p}\right\}$ are respectively the nodal displacements, velocity and nodal acceleration vectors and the time step $p$.

The numerical coupling between the two codes is achieved by using the transpiration method. This technique allows to take into account the displacement effect of the structure and to keep simultaneously a fixed domain for the fluid [10]. The main idea of this strategy is shown schematically in Fig. 1 . Here, $\Omega_{0}^{\mathrm{f}}$ and $\Omega_{0}^{\mathrm{s}}$ designate respectively the fluid and the structure reference domains, and $\Gamma_{0}^{\mathrm{s}}$ designates the fluid-structure interface.

As we can see, the new location of the fluid-structure interface $\Gamma_{0}^{\mathrm{s}}$ at the instant $t$ is $\Gamma^{\mathrm{s}}(t)$. The concept of transpiration method requires to keep $\Gamma_{0}^{\mathrm{s}}$ as fixed interface (immobile); the calculation of the velocity field and the dynamic loads is obtained by a first-order Taylor development at the interface of reference $\Gamma_{0}^{\mathrm{s}}$. When calculating the structure dynamic, a node which is initially in the position $x_{0}$ is displaced by a distance of $\delta x^{\mathrm{s}}\left(x_{0}, t\right)$. By neglecting the higher-order terms and for the inviscid flow case, the transpiration condition for the velocity can be reduced to the flowing simplified form on $\Gamma_{0}^{\mathrm{s}}[10]$ :

$$
u_{E} \cdot n_{0}=\delta \dot{x}^{\mathrm{s}} \cdot n-u_{E} \cdot\left(n-n_{0}\right)
$$

where $n_{0}$ and $n$ are the normal unit vectors to the wall in reference $\Gamma_{0}^{\mathrm{s}}$ and in deformed configurations $\Gamma^{\mathrm{s}}(t)$, respectively, and $\delta \dot{x}^{\mathrm{s}}$ is the speed of the flexible interface.

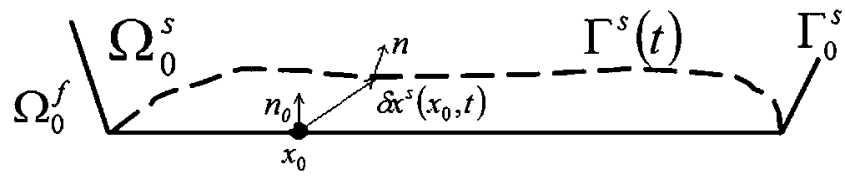

Fig. 1 The illustrated scheme for the law of transpiration [10]

\section{Results}

In order to compare the results issued from the numerical coupling to these of the aeroelastic stability models, 2D nozzle geometry has been chosen with fixed nodes at throat and at the exit of the nozzle divergent (Fig. 2). The material properties are summarised in Table 1.

The numerical coupling strategy consists as a first step to identify the initial position of the shock steady state for a rigid structure. Then, in second time, the structure is perturbed following to its first mode, for example. The aeroelastic analysis consists in recording the temporal evolution of the vertical displacements for a given node of the structure and then to compute the frequency values by using the fast Fourier transform (FFT). Figure 3 shows the evolution of the real part of the aeroelastic frequency versus shock wave position. As we can see, except the zone of the static instabilities, the results predicted by the numerical coupling are in general in good agreement with those issued from the model predictions. In the first quarter of divergent and for the region near the exit, the structure is stable as provided by the models. In addition, in the dynamic instability area, corresponding to the coalescence of modes 1 and 2, the coupled computation indicates that the temporal evolution of the normal structure displacement and their Fourier transform adopt the same behaviour in the region of flutter instability as shown in Fig. 4a. This evolution is harmonic having an increasing amplitude,

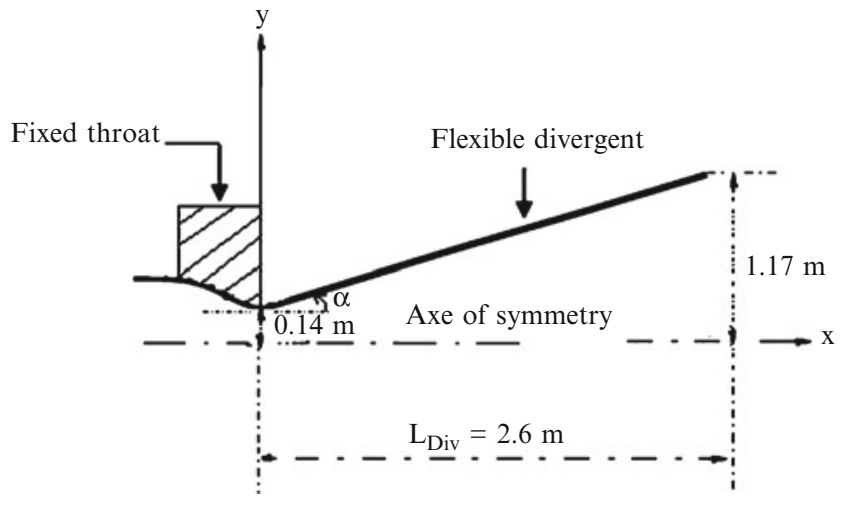

Fig. 2 Nozzle geometry for the studied test case

Table 1 Material properties (2D)

\begin{tabular}{l|l|l|l}
\hline $\begin{array}{l}\text { Young modulus } \\
\left(\mathrm{N} / \mathrm{m}^{2}\right)\end{array}$ & $\begin{array}{l}\text { Thickness } \\
h(\mathrm{~mm})\end{array}$ & $\begin{array}{l}\text { Poisson } \\
\text { coefficient }\end{array}$ & $\begin{array}{l}\text { Mass } \\
\text { density }\end{array}$ \\
\hline $2.2 \times 10^{11}$ & 10 & 0.0 & $2000 \mathrm{~kg} / \mathrm{m}^{3}$ \\
\hline
\end{tabular}


Fig. 3 Evolution of the aeroelastic frequencies versus shock separation position, comparison between the numerical results issued from the coupled computation and the stability models $\left(\alpha=22^{\circ}\right)$

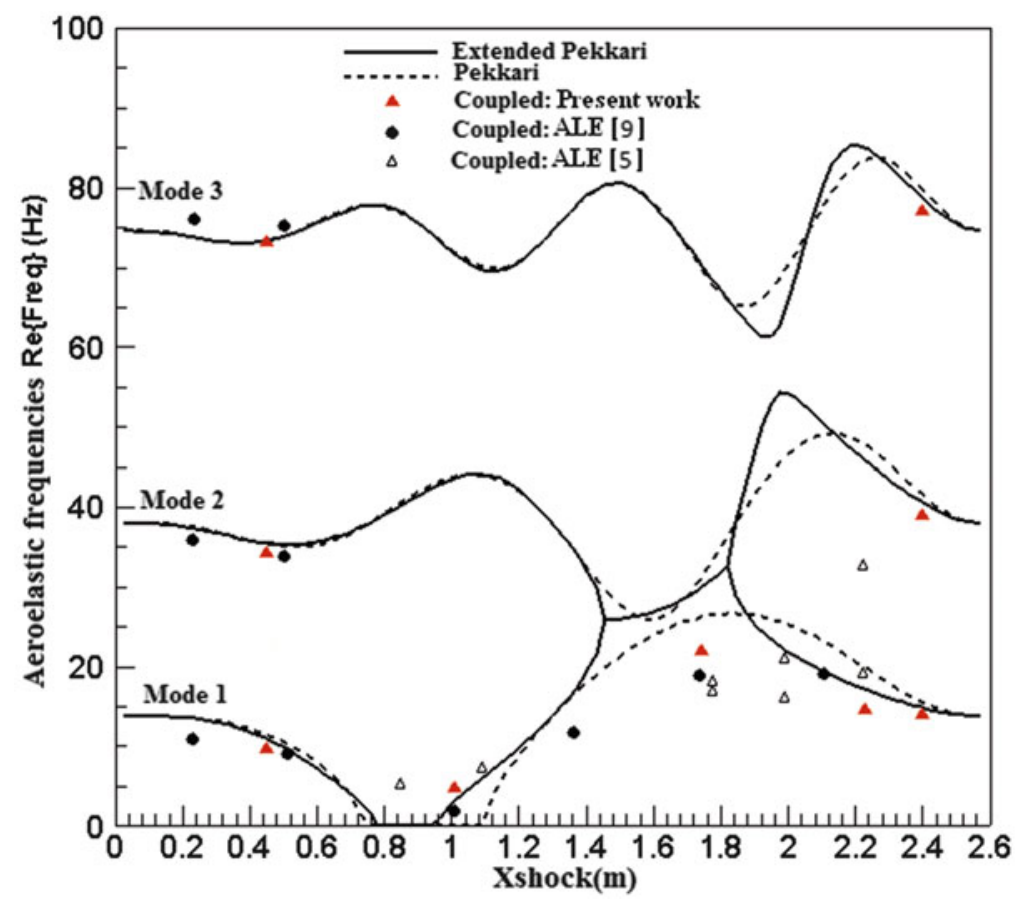

(a)

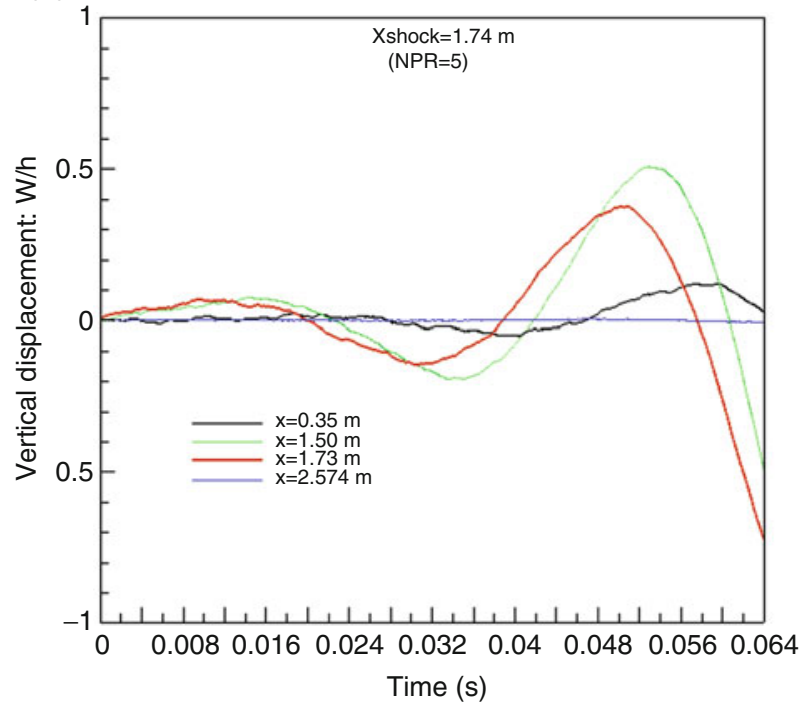

(b)

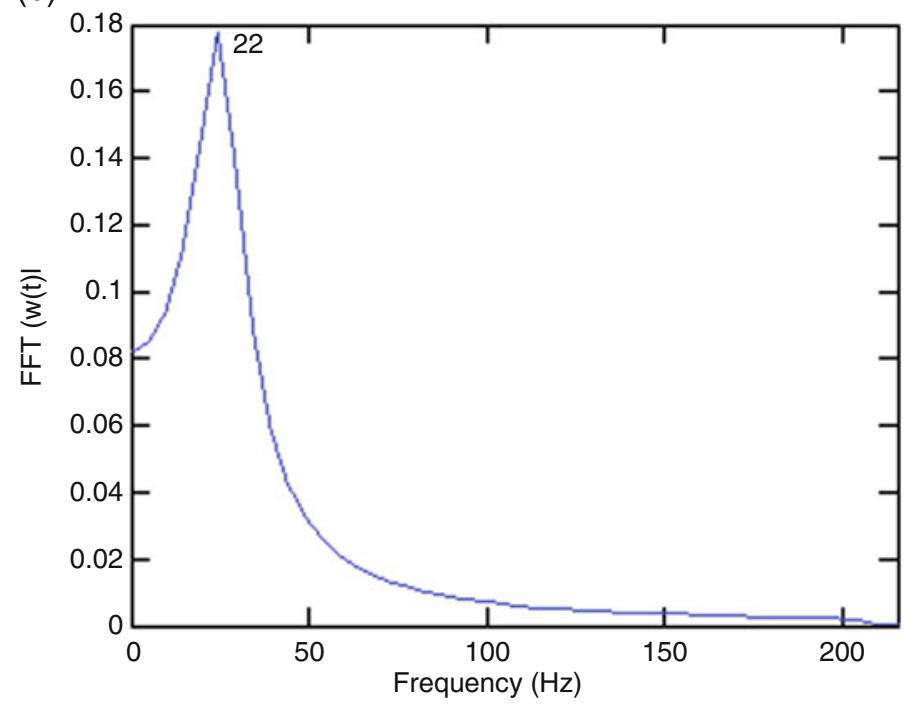

Fig. 4 Evolution of the vertical displacement (a) and its Fourier transform (b) for NPR $=5$

characterising the phenomenon of dynamic instability or flutter. This type of instability may lead to the structure failure and represents a major issue in industrial context.

\section{Conclusions}

In this study, the aeroelastic stability analysis for the overexpanded nozzles has been performed using the numerical coupling via the transpiration method. The results show that a linear approach for the small displacements is usually sufficient for the dynamic instabilities prediction. However, the ulterior evolution of the structure behaviour involves necessarily a nonlinear modelling, and the method of transpiration becomes inoperative.

\section{References}

1. Bekka, N.: Problématique des ondes de choc dans les tuyères supersoniques et leur interaction avec la structure. Ph.D. thesis, Université d'Evry Val d'Essonne (2014) 
2. Blades, E.L., Baker, M., Pray, C.L., Luke, E.A.: Fluid-structure interaction simulations of rocket engine side loads. SIMULIA Customer Conference (2012)

3. Garelli, L., Paz, R.R., Storti, M.A.: Fluid-structure interaction study of the start-up of rocket engine nozzle. J. Comput. Fluids 39, 1208-1218 (2010)

4. Lefrançois, E.: Numerical validation of a stability model for a flexible over-expanded rocket nozzle. Int. J. Numer. Methods Fluids 49, 349-369 (2005)

5. Lefrançois, E.: Modèle numérique de couplage fuide-structure pour l'étude des phénomènes aéroélastiques avec applications aux moteurs fusée. Ph.D. thesis, Université de Rouen (1998)

6. Lüdeke, H., Calvo, J.B., Filimon, A.: Fluid structure interaction at the ARIANE-5 Nozzle section by advanced turbulence models. In:
European Conference on Computational Fluid Dynamics, ECCOMAS CFD (2006)

7. Mouronval, A.-S.: Etude numérique des phénomènes aéroélastiques en aérodynamique supersonique. Application aux tuyères propulsives. Ph.D. thesis, INSA de Rouen (2004)

8. Mohammadi, B.: Fluid Dynamics Computation with NSC2KE. An User-Guide, Release 1.0, N RT-0164, INRIA (1994)

9. Piperno, S., Farhat, C.: Energy based design and analysis of staggered solvers for nonlinear transient aeroelastic problems. AIAA Paper 2000-1447 (2000)

10. Fernández-Varela, M.A.: Modèles simplifiés d'Interaction FluideStructure. Ph.D. thesis, Université IX Dauphine (2001) 